\title{
Quantitative evaluation of crossed
}

\section{cerebellar diaschisis, using voxel-based analysis of Tr-99m BCID brain SPECT'}

Anna Nocuń1, Joanna Wojczal², Hanna Szczepańska-Szerej², Marek Wilczyński ${ }^{3}$, Beata Chrapko'

${ }^{1}$ Department of Nuclear Medicine

${ }^{2}$ Chair and Department of Neurology

${ }^{3}$ Chair and Department of Vascular Surgery and Angiology

Medical University of Lublin, Poland

[Received 29 VI 2012; Accepted 28 XI 2012]

\section{Abstract}

BACKGROUND: In the recent literature there is no consensus regarding the relationships between crossed cerebellar diaschisis (CCD) with the primary lesion size, severity or location. Thus, the aim of the present study was to investigate relationship between the size and severity of cerebral lesions and CCD in patients with chronic stroke, using voxel-based analysis of Tc-99m ECD single-photon emission computed tomography (SPECT). MATERIAL AND METHODS: We retrospectively reviewed data of 57 patients with chronic ischemic lesions localized unilaterally in the cerebral hemisphere. SPECT evaluation was performed with the voxel-based analysis. The percentage inter-hemispheric asymmetry index (Al) and the volume of abnormal clusters of voxels $(\mathrm{CV})$ were ascertained for hypoperfusion in the supratentorial lesion and contralateral cerebellum.

RESULTS: CCD was present in $35.1 \%$ cases. In Group CCD $(+)$, the $\mathrm{CV}$ and $\mathrm{Al}$ of supratentorial hypoperfusion (median $128.1 \mathrm{ml}$ and $21.9 \%$, respectively) were significantly higher compared with Group $\operatorname{CCD}(-)$ (median $41.4 \mathrm{ml}$ and $18.0 \%$, respectively). Statistically significant correlation was found between $\mathrm{CV}$ of supratentorial and cerebellar perfusion defects $(r=0.4 ; p<0.05)$, between Al of supratentorial and cerebellar perfusion defects $(r=0.6 ; p<0.05)$ and between $C V$ of

Correspondence to: dr n. med. Anna Nocuń

Department of Nuclear Medicine

ul. Jaczewskiego 8, 20-954 Lublin, Poland

Tel/fax: (81) 7244339

e-mail: ampolak@o2.pl supratentorial defect and $\mathrm{Al}$ of cerebellar perfusion defects $(r=0.6 ; p<0.05)$.

CONCLUSIONS: Our data suggest, that in the chronic stage of stroke, the size and severity of the supratentorial lesion are determinants of $C C D$, correlating with the degree of cerebellar hypoperfusion.

KEY words: chronic stroke, crossed cerebellar diaschisis, single-photon emission computed tomography, voxel-based analysis

Nuclear Med Rev 2013; 16, 1: 31-34

\section{Background}

Different kinds of brain injuries: vascular, traumatic or tumors, can interrupt connections and impair neuronal impulses to other cerebral regions distant from the original lesion [1]. Brain areas receiving poor afferent signals decrease their metabolism, and subsequently perfusion. This unique phenomenon is referred to as deafferentation or diaschisis. The most commonly seen pattern of deafferentation, well-recognized after cerebral infarction is crossed cerebellar diaschisis (CCD), defined as matched depression of blood flow and metabolism in the cerebellar hemisphere contralateral to a supratentorial lesion [2]. The most likely mechanism underlying CCD is the interruption of the corticopontocerebellar pathway [2]. Such interruption is usually secondary to decreased cerebral perfusion, however it may be associated with supratentorial hyperperfusion after revascularization procedures [3, 4]

In the recent literature, based on the quantification of CCD with single-photon emission computed tomography (SPECT), positron emission tomography (PET), and magnetic resonance (MR) perfusion imaging, there is no consensus regarding the relationships between CCD with the primary lesion size, severity or location. The vast majority of the reports focus attention on acute and subacute phases of stroke [5-8], only few aim to elucidate these controversial issues in the chronic phase [9], when the CCD is already stabilized and when there is still no evidence of a direct correlation between supratentorial hypoperfusion volume and the degree of CCD [9]. 
Therefore, the purpose of the present study was to investigate relationship between the size and severity of cerebral lesions and CCD in patients presenting with chronic stroke, using voxel-based analysis of Tc-99m ECD brain SPECT.

\section{Material and methods}

\section{Patients}

We retrospectively reviewed data of 57 consecutive patients who underwent brain perfusion SPECT in our institution and were diagnosed with ischemic stroke $2-15$ months earlier. The selected sample consisted of 33 men and 24 women, aged 35-85 years (mean: $65 \pm 19.5$ years). CT or MR scans were performed in all participants in different medical centers. The ischemic lesions were localized unilaterally in the cerebral hemisphere. Patients with cerebellar abnormalities or bilateral cerebral pathology were excluded from the study.

\section{Data acquisition}

Brain perfusion SPECT was performed after intravenous injection of 99mTc-ethyl cysteinate dimmer (99mTc-ECD) (Department of Radiopharmaceuticals Production, Medical University in Lodz, Poland) at an activity of $740 \mathrm{MBq}$. Acquisition was carried out 20-30 min after radiotracer administration, using a rotating, double-head, large field of view gamma camera (Varicam, GE Medical Systems), equipped with low-energy, high-resolution collimators. The data were collected in a $128 \times 128$ matrix through $360^{\circ}$ rotation at $3^{\circ}$ intervals for $25 \mathrm{~s}$ per view. Reconstruction of transaxial slices was performed by filtered back projection (Metz filter power, 3.00; full width at half maximum, $10 \mathrm{~mm}$ ) with subsequent attenuation correction using the Chang method (attenuation coefficient 0.11).

\section{Data analysis}

SPECT scans were assessed semiquantitatively with Brain SPECT Quantification software (Compart Medical Systems, Poland). The method, which was described previously $[10,11]$, consists of the four major steps.

Step 1 - the patient study is interpolated to the same resolution $(2 \times 2 \times 2 \mathrm{~mm})$ as the anatomically standardized brain template (norm), provided by the manufacturer with the software.

Step 2 - the software automatically matches individual SPECT with the template. Such a transformation process, defined as spatial normalization, consists of scaling, shifting and rotation. It is associated with count normalization to the whole brain. The quality of the fusion of the patient study and the template is displayed and may be verified.

Step 3 - voxel-based analysis takes place. In each voxel, asymmetry index $(\mathrm{Al})$ is calculated, using the formula: $\mathrm{Al}=(\mathrm{A}-\mathrm{B}) /$ $/(A+B) / 2 \times 100 \%$, where $A$ and $B$ represent counts in the symmetrical voxels of the left and right hemisphere.

Step 4 - cluster analysis is performed. Abnormalities are discerned by a 3D region-growing algorithm which compares voxels in the image to the corresponding limits (cut-off values). Cut-off values that can be set by the user in a program configuration were set as follows: Al higher than 10\% [12] in a cluster volume greater than $10 \mathrm{ml}$. Contiguous regions are identified for which these conditions are met. Clusters of abnormal voxels are listed and reported with respect to the absolute cluster size in ml-cluster volume (CV) and severity (the mean percentage of $\mathrm{Al}$ in the cluster region).

Semiquantitative analysis allowed for evaluation of hypoperfusion in the supratentorial lesion and contralateral cerebellum in terms of the size (CV) and severity (Al). Example of cluster analysis in a patient wit CCD is shown on Figure 1.

\section{Statistical analysis}

The data set was not normally distributed (Shapiro-Wilk test), therefore the non parametric approach was applied. Data are expressed as median and interquartile range (25th and 75th percentiles). Comparison between parameters of supratentorial hypoperfusion (CV and $\mathrm{Al}$ ) in the patients with and without CCD: Group $\mathrm{CCD}(+)$ and Group $\mathrm{CCD}(-)$ was performed by the Mann- Whitney test (unpaired variables). The correlations between parameters of supratentorial and cerebellar perfusion defects in Group CCD(+) were assessed by means of Spearman's Rank Correlation Test. The P-value equal or less than 0.05 was considered statistically significant. Statistical analysis was performed using the software STATISTICA (StatSoft, Poland).

\section{Results}

CCD was present in 20 cases (35.1\%). In these cases, values of CV and Al in hypoperfused cerebellum were: CV ( median $24.5 \mathrm{ml}$, range $10.2-40.9 \mathrm{ml})$;

Al (median 17.7\%, range 12.1-24.3\%); the values of CV and Al obtained in supratentorial lesions are summarized in Table 1. In Group CCD(+), CV and Al (median $128.1 \mathrm{ml}$ and 20.9\%, respectively) were significantly higher compared with Group CCD(-) (median $41.4 \mathrm{ml}$ and 18.0\%, respectively). Correlations between the values of $\mathrm{Al}$ and CV in Group CCD(+) are presented in Table 2. Statistically significant correlations were found between CV of supratentorial and cerebellar perfusion defects (correlation coefficient $=0.4 ; p<0.05$ ), between Al of supratentorial and cerebellar perfusion defects (correlation coefficient $=0.6 ; p<0.05$ ) and between $\mathrm{CV}$ of supratentorial defects and $\mathrm{Al}$ of cerebellar perfusion defects (correlation coefficient $=0.6 ; p<0.05$ ). There was no significant correlation between $\mathrm{Al}$ of supratentorial defects and $\mathrm{CV}$ of cerebellar perfusion defects.

\section{Discussion}

The incidence of CCD in our clinical sample (35.1\%) is within the wide range of the values obtained by other authors: 15.6\% [5], $31 \%$ [13], 39.8\% [12], 46\% [14]. Finding that CCD predominantly appears in larger supratentorial perfusion defects corroborate the majority of previous works $[5,14]$. The massive infarction is more likely to include important functional structures and pathways that contribute to CCD [7]. In the present study, cerebellar Al values correlated with both the size and asymmetry of cerebral hypoperfusion, which respectively represent the number of impaired neurons in the cortico-ponto-cerebellar pathway and severity of the functional damage. To the best of our knowledge, such correlations obtained with voxel-based analysis of SPECT in the chronic stage of stroke were not reported so far in the available literature.

In the PET study of acute ischemia [6], the volume of cerebral hypoperfusion correlated with CCD values before thrombolysis (that 

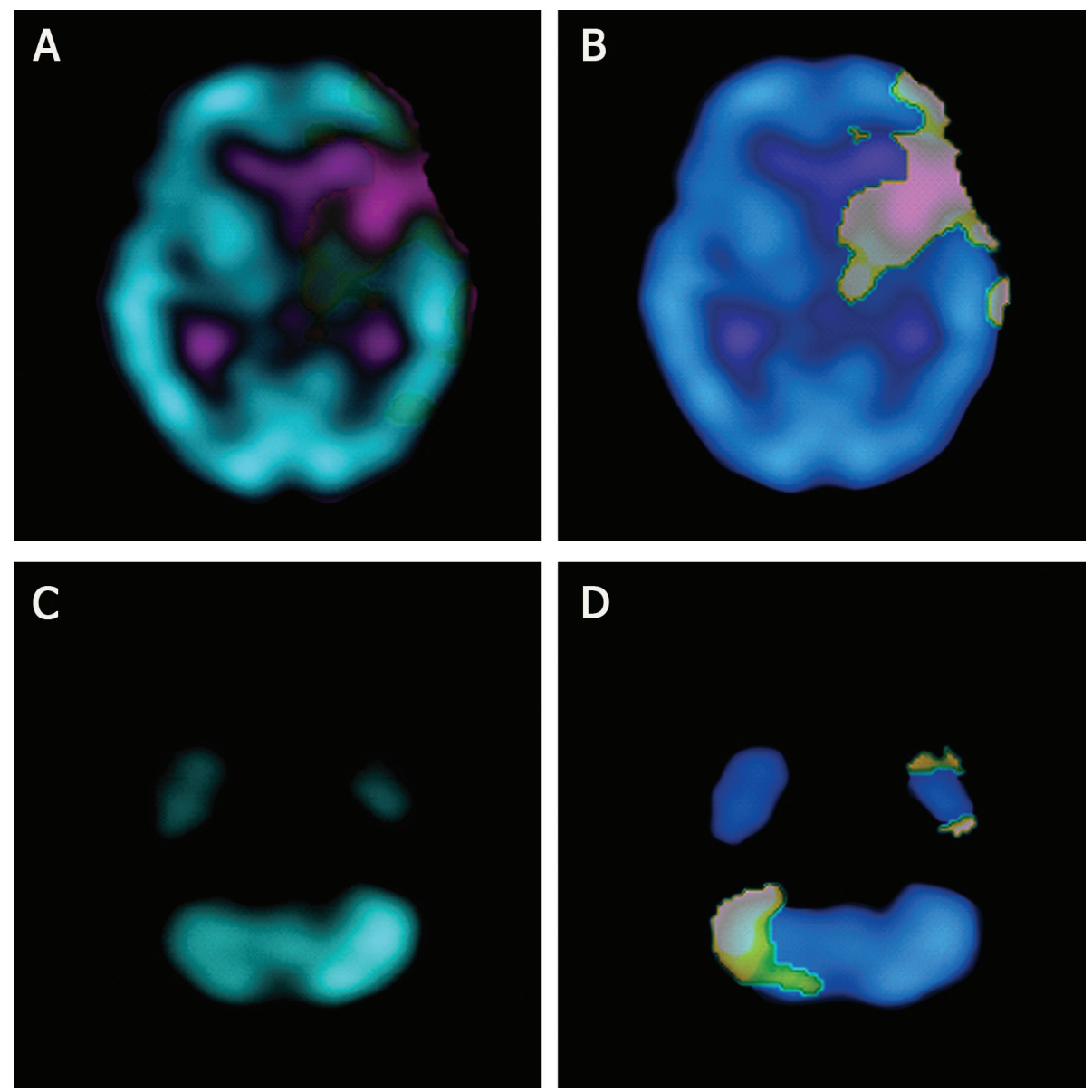

Figure 1. $C C D$ in a patient with the infarction in the left cerebral hemisphere. A. Cerebral hypoperfusion. B. Cluster analysis of the supratentorial lesion $(\mathrm{CV}=127 \mathrm{ml}, \mathrm{Al}=23.5 \%)$. C. Hypoperfusion in the right cerebellar hemisphere. $\mathbf{D}$. Cluster analysis of the subtentorial hypoperfusion $(\mathrm{CV}=37.9 \mathrm{ml}, \mathrm{Al}=20.1 \%)$

Table 1. Quantitative evaluation of supratentorial perfusion defects

\begin{tabular}{|c|c|c|c|c|c|c|c|}
\hline \multirow[b]{2}{*}{ Group } & \multirow[b]{2}{*}{$\mathrm{n}$} & \multicolumn{3}{|c|}{ Cluster volume (CV) [ml] } & \multicolumn{3}{|c|}{ Asymmetry index (Al) (\%) } \\
\hline & & Range & Median & $25-75 \%$ & Range & Median & $25-75 \%$ \\
\hline$\overline{\mathrm{CCD}(+)}$ & 20 & $10.9-277$ & 128.1 & $79.5-176.2$ & $12.1-29.3$ & 20.9 & $19.3-23.9$ \\
\hline $\operatorname{CCD}(-)$ & 37 & $10.2-152$ & 41.4 & $22.0-101.0$ & $11.5-31.3$ & 18.0 & $17.0-19.9$ \\
\hline P-value & & & 0.0001 & & & 0.007 & \\
\hline
\end{tabular}

Table 2. Correlations in Group $\operatorname{CCD}(+)$

\begin{tabular}{lllc}
\hline & Correlation & & \\
\hline Supratentorial perfusion defects & Cerebellar perfusion defects & Correlation coefficient & P-value \\
\hline Cluster Volume (CV) & Cluster Volume (CV) & 0.4 & $<0.05$ \\
& Asymmetry Index (Al) & 0.6 & $<0.05$ \\
Asymmetry Index (Al) & Cluster Volume (CV) & & Not significant \\
& Asymmetry Index (Al) & 0.6 & $<0.05$ \\
\hline
\end{tabular}

is within 3 hours after symptom onset) and 3 hours after thrombolysis, but not in the later measurements performed 24 hours and 14 days after thrombolysis. At these later time points, CCD was disconnected from supratentorial lesion, but was closely associated with the clinical outcome. As authors conclude, this "disconnection" means that cerebellar asymmetry is not a simple mirror of cerebral perfusion, but contains additional information, which may be valuable in the clinical practice. Interestingly, correlation between asymmetry of cerebral and cerebellar hypoperfusion was not statistically significant in acute stage of stroke. This association may appear 
later, when the functional impairment caused by the destruction of a cortical region is already stabilized.

The results of SPECT/MR study [7] of acute and subacute stroke showed, that CCD is determined by infarction volume and location. The number of papers emphasize the role of supratentorial lesion location as the decisive factor for CCD occurrence. The phenomenon is reported to be more frequent in the infarcts involving postcentral and supramarginal regions [12], frontoparietal lobes, basal ganglia and internal capsule [15]

Scarcity of our clinical sample did not enable investigation of relation between hypoperfusion location and CCD. Noteworthy is the fact, that the smallest infarct in our Group $\mathrm{CCD}(+)$ was localized in the thalamus, which is a collection of nuclei acting as a highly sophisticated relay station between the cerebral cortex and cerebellum. Despite low volume, thalamic damage was previously observed to result in functional cerebro-cerebellar disconnection [5].

Based on the fiber projection of the cerebral cortex to the cerebellar deep nuclei, CCD may be limited to one of the three longitudinal zones: medial, intermediate and lateral [7]. Therefore, Al quantification from the regions of interest (ROls) covering the whole cerebellum can lead to the controversial results [7]. Instead of conventional ROls drawn over one slice of the cerebellum, voxel- based approach was employed in the present work. This newer image processing technique is automatic and 3-dimensional. It gives the fast and objective analysis of the entire cerebellum instead of one slice. In addition to more precise calculation of $\mathrm{Al}$, the voxel- based software allowed us to evaluate the size of cerebellar hypoperfusion (median $24.5 \mathrm{ml}$, range $10.2-40.9 \mathrm{ml}$ ) and to correlate this volume with supratentorial hypoperfusion values. Obtained results suggest relationship between CCD volume with infarction volume $(p<0.05)$, but not with severity of cerebral hypoperfusion $(p>0.05)$. This finding implies that CCD is only in part a reflection of supratentorial disturbances.

The essential yet controversial issue - the clinical value of CCD - was not explored in our study due to its retrospective character and lack of the homogenous scoring system of neurological symptoms in patients referred from different medical departments. Recent papers highlight poor improvement of patients with CCD in acute stroke [6-8], indicating that CCD may be a valuable prognostic factor in this phase of the disease. In the recent PET study [9], performed in the chronic stage 7-76 months after stroke, significant correlations were found between clinical scales and the CCD values. As authors conclude, CCD is a disease biomarker that can be a measurement of the functional deficit resulting from a stroke in routine stroke care.

\section{Conclusions}

Our data suggest that in the chronic stage of stroke, the size and severity of the supratentorial lesion are determinants of CCD, correlating with the degree of cerebellar hypoperfusion.

The software with voxel-based analysis of brain perfusion SPECT makes a useful tool for CCD evaluation.

\section{References}

1. Flores LG, Futami S, Hoshi H et al. Crossed cerebellar diaschisis: analysis of iodine-123-IMP SPECT imaging. J Nucl Med 1995; 36: 399-402.

2. Catafau AM. Brain SPECT in clinical practice. Part I: perfusion. J Nucl Med 2001; 42: 259-271.

3. Ogasawara K, Kobayashi M, Suga $Y$ et al. Significance of postoperative crossed cerebellar hypoperfusion in patients with cerebral hyperperfusion following carotid endarterectomy: SPECT study. Eur J Nucl Med Mol Imaging 2008; 35: 146-152

4. Hokari M, Kuroda S, Simoda Y et al. Transient crossed cerebellar diaschisis due to cerebral hyperperfusion following surgical revascularization for moyamoya disease. Neurol Med Chir 2012; 52: 350-353.

5. Lin DD, Kleinman JT, Wityk RJ et al. Crossed cerebellar diaschisis in acute stroke detected by dynamic susceptibility contrast MR perfusion imaging. AJNR Am J Neuroradiol 2009; 30: 710-715.

6. Sobesky J, Thiel A, Ghaemi M et al. Crossed cerebellar diaschisis in acute human stroke: a PET study of serial changes and response to supratentorial reperfusion. J Cereb Blood Flow Metab 2005; 25: 1685-1691.

7. Liu Y, Karonen JO, Nuutinen J, Vanninen E, Kuikka JT, Vanninen RL. Crossed cerebellar diaschisis in acute ischemic stroke: a study with serial SPECT and MRI. J Cereb Blood Flow Metab 2007; 27: 1724-1732.

8. Takasawa M, Watanabe M, Yamamoto S et al. Prognostic value of subacute crossed cerebellar diaschisis: single-photon emission CT study in patients with middle cerebral artery territory infarct. AJNR Am J Neuroradiol 2002; 23: 189-193.

9. Szilágyi G, Vas A, Kerényi L, Nagy Z, Csiba L, Gulyás B. Correlation between crossed cerebellar diaschisis and clinical neurological scales. Acta Neurol Scand 2012; 125: 373-381.

10. Nocuń A, Wilczyński M, Wroński J, Chrapko B. Usefulness of 99mTc-ECD brain SPECT with voxel-based analysis in evaluation of perfusion changes early after carotid endarterectomy. Med Sci Monit 2011; 17: 297-303.

11. Nocuń A, Szajner M, Obszańska $K$ et al. Changes of brain perfusion after endovascular embolization of intracranial arteriovenous malformations visualized by 99mTc-ECD SPECT. Nucl Med Commun 2008; 29: 1073-180.

12. Komaba Y, Mishina M, Utsumi K, Katayama Y, Kobayashi S, Mori O. Crossed cerebellar diaschisis in patients with cortical infarction: logistic regression analysis to control for confounding effects. Stroke 2004; 35: 472-476.

13. Jeon YW, Kim SH, Lee JY et al. Dynamic CT perfusion imaging for the detection of crossed cerebellar diaschisis in acute ischemic stroke. Korean J Radiol 2012; 13: 12-19

14. Infeld B, Davis SM, Lichtenstein M, Mitchell PJ, Hopper JL. Crossed cerebellar diaschisis and brain recovery after stroke. Stroke 1995; 26: 90-95.

15. Kim SE, Choi CW, Yoon BW et al. Crossed-cerebellar diaschisis in cerebral infarction: technetium-99m-HMPAO SPECT and MRI. J Nucl Med 1997; 38: $14-19$ 